\title{
Phonons in twisted transition-metal dichalcogenide bilayers: Ultrasoft phasons and a transition from a superlubric to a pinned phase
}

\author{
Indrajit Maity, Mit H. Naik, Prabal K. Maiti, H. R. Krishnamurthy, and Manish Jain $\odot^{*}$ \\ Centre for Condensed Matter Theory, Department of Physics, Indian Institute of Science, Bangalore 560012, India
}

(Received 4 June 2019; revised manuscript received 9 January 2020; accepted 5 February 2020; published 18 March 2020)

\begin{abstract}
The tunability of the interlayer coupling by twisting one layer with respect to another layer of two-dimensional materials provides a unique way to manipulate the phonons and related properties. We refer to this engineering of phononic properties as twistnonics. We study the effects of twisting on low-frequency shear modes (SMs) and layer breathing modes in a transition-metal dichalcogenide (TMD) bilayer using atomistic classical simulations. We show that these low-frequency modes are extremely sensitive to twisting and can be used to infer the twist angle. We find ultrasoft phason modes (frequency $\lesssim 1 \mathrm{~cm}^{-1}$, comparable to acoustic modes) for any nonzero twist, corresponding to an effective translation of the moiré lattice by relative displacement of the constituent layers in a nontrivial way. Unlike the acoustic modes, the velocity of the phason modes are quite sensitive to the twist angle. Also, high-frequency SMs appear for small twist angles, identical to those in stable bilayer TMD $\left(\theta=0^{\circ}\right.$ or $\left.60^{\circ}\right)$, due to the overwhelming growth of stable stacking regions in relaxed twisted structures. Our study reveals the possibility of an intriguing $\theta$-dependent superlubric to pinning behavior and of the existence of ultrasoft modes in all two-dimensional materials.
\end{abstract}

DOI: 10.1103/PhysRevResearch.2.013335

\section{INTRODUCTION}

Twisting one layer of a bilayer system with respect to another provides a unique degree of freedom for tuning the properties of two-dimensional (2D) materials. For example, in the case of bilayer graphene, twisting leads to (a) structural changes, such as the observation of topological point defects, domain walls, and layer buckling [1-8], (b) significant change in electronic properties including superconductivity at magic twist angles [9-23], and (c) superlubricity, a state of ultralow friction [24-26]. An important facet of twisting is the evolution of low-frequency vibrational modes, which has largely remained unexplored. Since the low-frequency modes are solely determined by interlayer coupling and are accessible in Raman measurements, they provide a direct nondestructive probe of the interlayer interaction [27-30]. The existing theoretical reports on the evolution of vibrational modes in twisted structures are restricted to large twist angles and use the Lennard-Jones potential [31] to describe the interlayer interaction, which is insufficient for capturing the stackingdependent energetics [32]. Although existing experimental studies $[29,30,33]$ have explored small twist angles, they can only probe Raman active modes with frequencies greater than $10 \mathrm{~cm}^{-1}$.

\footnotetext{
*mjain@iisc.ac.in
}

Published by the American Physical Society under the terms of the Creative Commons Attribution 4.0 International license. Further distribution of this work must maintain attribution to the author(s) and the published article's title, journal citation, and DOI.
In this work we computationally investigate the effects of twisting on low-frequency shear modes (SMs) and layer breathing modes (LBMs) in bilayer $\mathrm{MoS}_{2}$, a prototypical transition-metal dichalcogenide (TMD). Relative in-plane and out-of-plane displacements of the constituent layers give rise to SMs and LBMs, respectively. The coexistence of several stackings in the moiré superlattice (MSL) that results from the twist leads to inhomogeneous interlayer coupling. As a consequence, the low-frequency modes mix and become quite sensitive to twisting. Our calculations show the existence of ultrasoft phason modes and large variation in LBM frequencies and the appearance of multiple LBMs and high-frequency SMs in twisted bilayer (TBL) $\mathrm{MoS}_{2}$. Moreover, we find that the velocity of the phason modes is quite sensitive to the twist angle. These observations are generic to TMDs and we confirm our results for $\mathrm{MoSe}_{2}$ as well. The domain walls and point defects present in twisted structures that are inevitable consequences of structural relaxation are likely to influence the electronic properties [34-37].

The paper is organized in the following manner. In Sec. II we detail the methods used to perform molecular dynamics simulations and computation of phonon frequencies. In Sec. III A we show the effects of relaxation on the rigidly twisted structures using an interlayer separation landscape. In Sec. III B we discuss mode mixing due to the existence of multiple coexistent stackings. In Sec. IIIC we show the twist angle dependence of shear and layer breathing modes. In Sec. III D we discuss the origin of phason modes in twisted structures and its role in determining frictional properties of the system. In Sec. IV we show the twist angle dependence of phonon frequencies of twisted bilayer $\mathrm{MoSe}_{2}$ and discuss experiments that can be used to test our predictions. We 
(a)

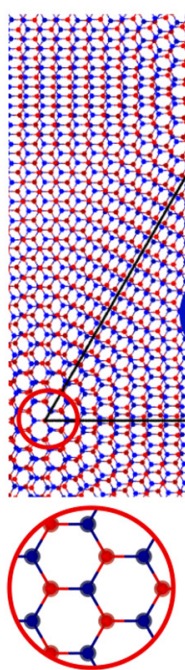

AA

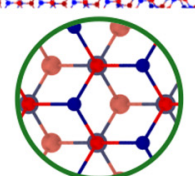

$A B$

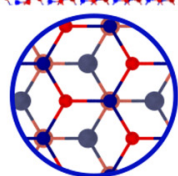

BA (b)

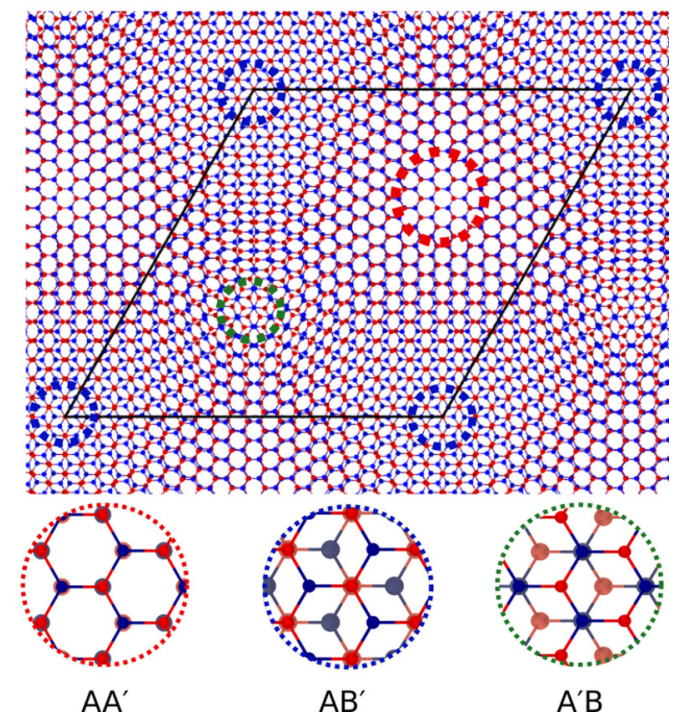

FIG. 1. Relaxed twisted bilayer of $\mathrm{MoS}_{2}$ and the coexistence of multiple high-symmetry stacking regions for (a) $\theta=2.9^{\circ}$ and (b) $\theta=$ $57.1^{\circ}$. The high-symmetry stacking regions are marked with solid and dashed circles. Mo atoms of bottom (top) layer are depicted as large (small) in size and with faded (dark) red color. Similarly, we use blue for S atoms.

also discuss the effects of manipulation of phonons on other properties such as specific heat. In Sec. V we summarize our results.

\section{SIMULATION DETAILS}

We use the TWISTER code [34] to create the MSL of bilayer $\mathrm{MoS}_{2}$ with several commensurate twist angles $1^{\circ}<$ $\theta<59^{\circ}$. The rigidly twisted structures are relaxed using LAMMPS [38-40] with the Stillinger-Weber and KolmogorovCrespi potentials to capture the intralayer $[41,42]$ and interlayer interactions of TBL $\mathrm{MoS}_{2}[32,43]$, respectively. The Kolmogorov-Crespi parameters used in our calculations correctly reproduce the interlayer binding energy landscape, obtained using density functional theory. The atomic relaxations produced using these parameters are in excellent agreement with relaxations performed using density functional theory. Moreover, the computed SM and LBM frequencies of the most stable stacking of bilayer of TMDs are in good agreement with experiments. The interlayer potential consists of two types of interaction: (i) nearest-neighbor S-S interaction and (ii) next-nearest-neighbor Mo-S interaction. All the parameters reported in Ref. [32] should produce similar results. We use modified PHONOPY [44,45] code to compute the zero-temperature vibrational spectra of the relaxed TBL $\mathrm{MoS}_{2}$. While relaxing the rigidly twisted structures, the force tolerance for any atom along any direction was set to be $10^{-6} \mathrm{eV} / \AA$.

Independently, we also compute the low-frequency modes from the power spectra of mode-projected velocity autocorrelation function (MVACF) from classical molecular dynamics simulations with periodic boundary conditions in the canonical ensemble using the Nosé-Hoover thermostat in LAMMPS. We equilibrate the system in the canonical ensemble for $\sim 150-300 \mathrm{ps}$ at $T=300 \mathrm{~K}$. The supercell contains 32 MSLs in order to correctly capture all the anharmonic effects.
Typically, we collect velocities of all atoms in the production run ( $480 \mathrm{ps,} \mathrm{in} N V E$ ensemble) every 20 time steps (one time step equals $1 \mathrm{fs}$ ). For computational efficiency, the 480-ps trajectory is divided into six parts, each containing $80 \mathrm{ps}$. The power spectra of the MVACF projects the full phonon spectra to a particular branch for any $\vec{q}$. The time-averaged MVACF at momentum $\vec{q}$ and polarization $s$ is defined as [46]

$$
\left\langle V_{\vec{q}, s}(0) V_{\vec{q}, s}^{*}(t)\right\rangle=\lim _{\tau \rightarrow \infty} \frac{1}{\tau} \int_{0}^{\tau} V_{\vec{q}, s}\left(t^{\prime}\right) V_{\vec{q}, s}^{*}\left(t+t^{\prime}\right) d t^{\prime},
$$

with

$$
V_{\vec{q}, s}(t)=\sum_{j=1}^{N_{t}} \vec{v}_{\vec{q}}^{j}(t) \cdot \hat{e}_{\vec{q}, s}^{j},
$$

where $j$ denotes the atom type in the unit cell and $\hat{e}_{\vec{q}, s}$ denotes the eigenvector. The mass-weighted momentum-projected velocities are defined as

$$
\vec{v}_{\vec{q}}^{j}(t)=\sqrt{m_{j}} \sum_{k} e^{-i \vec{q} \cdot \vec{r}_{j k}(t)} \vec{v}_{k},
$$

where $\vec{r}_{j k}$ are the atomic coordinates, $k$ denotes atoms belonging to particle type $j$, and $m_{j}$ denotes atomic mass. To compute the MVACF, we use the definition of an unbiased estimator [47]. Finally, we use a fast Fourier transform to compute the power spectra. Instead of computing the MVACF for each eigenmode in the MSL, we use bilayer (BL) $\mathrm{MoS}_{2}$ SMs and LBM eigenvectors to compute the MVACF of the superlattice.

\section{RESULTS}

A TBL $\mathrm{MoS}_{2}$ is composed of different high-symmetry stacking regions, which are different as $\theta \rightarrow 0^{\circ}$ and $\theta \rightarrow 60^{\circ}$ due to sublattice symmetry breaking (Fig. 1). For $\theta \rightarrow 0^{\circ}$ there are two unique high-symmetry stacking regions $A A$ (Mo and S of the top layer are directly above Mo and S of 
the bottom layer, respectively) and $A B$ (Bernal stacking with Mo of the top layer directly above $S$ of the bottom layer, equivalent to $B A$, also referred to as $3 R$ ). For $\theta \rightarrow 60^{\circ}$ there are three unique high-symmetry stacking regions $A A^{\prime}$ (Mo and $\mathrm{S}$ of the top layer are directly above $\mathrm{S}$ and Mo of the bottom layer, or $2 \mathrm{H}$ ), $A B^{\prime}$ (Bernal stacking with Mo of the top layer directly above Mo of the bottom layer), and $A^{\prime} B$ (Bernal stacking with $\mathrm{S}$ of the top layer directly above $\mathrm{S}$ of the bottom layer) [29]. Among these high-symmetry stackings $A B\left(A A^{\prime}\right)$ is the most stable with SM frequency $\sim 21 \mathrm{~cm}^{-1}$, whereas $A A$ $\left(A^{\prime} B\right)$ is unstable with strong imaginary SM frequency. Due to the difference in binding energies of different stackings [consequently, stability and interlayer separation (ILS)], upon relaxing the MSL the more stable stacking regions increase in area. The signatures of the growth of the stable stacking regions with $\theta$ are inherently embedded in the ILS landscape.

\section{A. Relaxation: Interlayer separation landscape}

For the calculation of vibrational properties, i.e., perturbation with respect to the ground state, the relaxation of the twisted structure is necessary to obtain a suitable ground state. The incorporation of relaxation effects are essential to compute any phonon frequencies. In order to illustrate this, we have computed the phonon frequencies of the unrelaxed twisted bilayer of $\mathrm{MoS}_{2}$ for $\theta=1.9^{\circ}$. We find large imaginary frequencies at the $\Gamma$ point in the phonon dispersion (23 unstable phonon modes with imaginary frequencies ranging in magnitude from $10 \mathrm{~cm}^{-1}$ to $45 \mathrm{~cm}^{-1}$ ). Most of these phonon modes also have imaginary frequencies along high-symmetry directions of the Brillouin zone. These imaginary frequencies denote structural instability of the rigidly twisted bilayer of $\mathrm{MoS}_{2}$. Upon relaxation, the atoms within the twisted structures reorganizes themselves and stabilizes the structure. Such atomic reconstruction removes the unstable phonon modes in the phonon dispersion. Due to this, we always compute phonon dispersion using relaxed structures.

The relaxation of the rigidly twisted structures involves straining and buckling of each constituent layers. In order to demonstrate the consequences of relaxation, in Fig. 2 we show the $\theta$ dependence of the ILS landscape and ILS av $_{\text {. We }}$ can identify the high-symmetry stacking regions in the ILS landscape for $\theta \rightarrow 0^{\circ}$ [Fig. 2(a)]: alternate triangles with the least ILS ( $A B$ and $B A$, deep blue) and red circles with the maximum ILS $(A A)$. The $A B$ and $B A$ regions grow equally for $\theta \rightarrow 0^{\circ}$ since they are degenerate in energy. Six domain walls (light blue lines) meet at the "centers," where $A A$ stacking (topological point defect) regions are located, similar to what happens in twisted bilayer graphene (TBLG) $[1,2,5,6]$. The ILS landscape for $\theta \rightarrow 60^{\circ}$, on the other hand [Fig. 2(b)], is very different due to sublattice symmetry breaking. The $A A^{\prime}$ regions (Reuleaux-triangle-like) grow overwhelmingly due to their lower binding energy. Six curved domain walls meet at the centers, where $A^{\prime} B$ (red circles, maximum ILS) stacking regions are located. The generalized stacking fault energy in conjunction with continuum theory is also shown to predict similar in-plane features [48] for TBL $\mathrm{MoS}_{2}$. It is interesting to note that both the length and the shape of domain walls can be tuned with twists as $\theta \rightarrow 60^{\circ}$. Figures 2(c) and 2(d) capture $\theta$ dependence of ILS $_{\mathrm{av}}, \mathrm{ILS}_{\min }$, and $\mathrm{ILS}_{\max }$ of
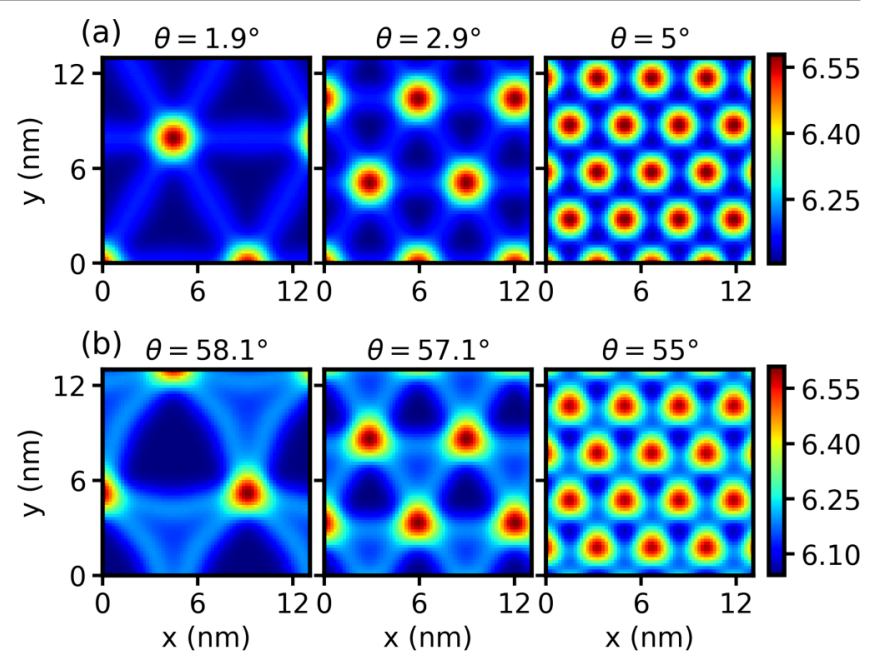

(c)

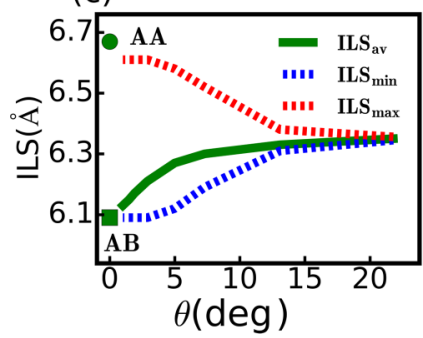

(d)

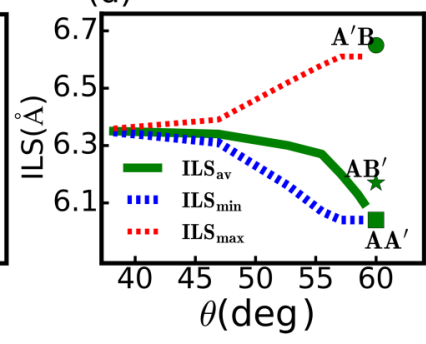

FIG. 2. Evolution of the ILS landscape and its average (in angstroms) with twist angle in $\mathrm{TBL} \mathrm{MoS}_{2}$. The in-plane ( $x$ and $y$ ) distances are in nanometers.

the MSL. For large twists $\left(13^{\circ}<\theta<47^{\circ}\right)$, the absence of any extended ideal high-symmetry stacking regions leads to $\theta$-independent behavior of ILS $_{\mathrm{av}}, \mathrm{ILS}_{\mathrm{min}}$, and $\mathrm{ILS}_{\mathrm{max}} ; \mathrm{ILS}_{\min }$ and $\mathrm{ILS}_{\max }$ saturate for $\theta<3^{\circ}$ and $\theta>57^{\circ}$, although $\mathrm{ILS}_{\mathrm{av}}$ does not saturate in this limit due to the presence of $A A$ or $A^{\prime} B$ and domain walls.

\section{B. Inhomogeneous interlayer coupling}

As a whole, twisting affects the phonon band structure in two ways. First, the shrinking of the Brillouin zone gives rise to folded phonon modes. Second, as there are multiple stackings in the MSL, the interlayer coupling is inhomogeneous, which leads to mode mixing among in-plane and out-of-plane modes. Relaxation of the rigidly twisted structures further changes this mode mixing and stabilizes the structure (the SM frequency of unrelaxed TBL $\mathrm{MoS}_{2}$ is strongly imaginary, implying instability). In order to separate the effects due to only zone folding and the mixing of modes due to the presence of multiple stacking, we compute $A^{i j}=\left|\left\langle\psi_{\mathrm{BL}}^{i} \mid \psi_{N I}^{j}\right\rangle\right|^{2}$ projections of bilayer eigenmodes $\left|\psi_{\mathrm{BL}}^{i}\right\rangle$ onto individual layer modes $\left|\psi_{N I}^{j}\right\rangle$ at $\Gamma$ for both untwisted and twisted structures [Figs. 3(a) and 3(b)]. The untwisted and twisted structures are of the same dimensions. The projections would have been identical if the zone-folding effects were the only factor determining phonons in twisted structures. It is clear from the figure that inhomogeneity in the interlayer coupling in the twisted structure leads to greater mode mixing. The comparison of mode mixing between untwisted and twisted structures is essential as the mixing strongly depends on the twist angle and produces 

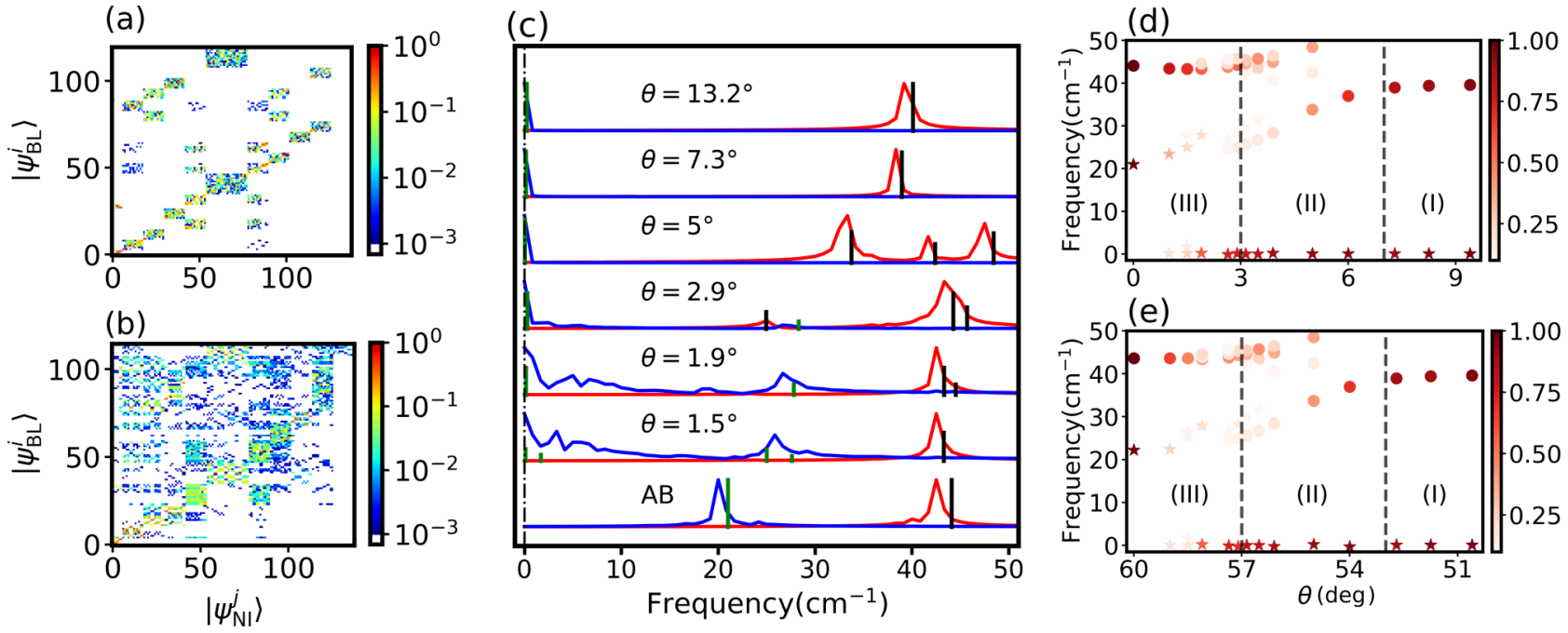

FIG. 3. Inhomogeneity in the interlayer coupling in the MSL and the evolution of low-frequency modes. Here $A^{i j}$ (see the text for definition) is plotted for (a) untwisted and (b) twisted $\left(\theta=57.1^{\circ}\right.$ ) bilayers of the same dimensions for modes with $\omega<50 \mathrm{~cm}^{-1}$. (c) SM (green) [LBM (black)] frequencies at $T=0 \mathrm{~K}$ marked as vertical lines with height proportional to $p^{\mathrm{SM}}\left(p^{\mathrm{LBM}}\right)$. Also shown are the power spectra of MVACF (with highest peak normalized to 1 ) at $T=300 \mathrm{~K}$. (d) SM and (e) LBM frequencies at $T=0 \mathrm{~K}$ with the color bar indicating $p^{\mathrm{SM}}$ and $p^{\mathrm{LBM}}$.

nontrivial effects as we illustrate below. This observation is also crucial, as it invalidates the usage of a simple linear chain model to compute SM and LBM frequencies.

\section{Shear and layer breathing modes}

Next we focus on the effects of twisting on the SM and LBM frequencies with (at $T=300 \mathrm{~K}$ ) and without thermal fluctuations $(T=0 \mathrm{~K})$. While computing the MVACF (for $T=300 \mathrm{~K}$ ) we use the SM and LBM eigenvectors of $\mathrm{BL}$ $\mathrm{MoS}_{2}$. As discussed above, due to mode mixing, the eigenvectors of the twisted structures can be composed of several normal modes of the MSL. Any nondegenerate eigenmode involving relative displacements of the layers of the MSL should appear as a distinct peak in the power spectra of the MVACF. In order to compare with finite- $T$ results we also project the eigenmodes of the MSL onto the $\mathrm{BL} \mathrm{MoS}_{2} \mathrm{SM}$ and LBM eigenvectors, $p=\left|\left\langle\hat{e}_{\mathrm{MSL}} \mid \hat{e}_{\mathrm{BL}}\right\rangle\right|^{2}$ at $T=0 \mathrm{~K}$ [Fig. 3(c)]. It should also be noted that multiple zone-folded modes from SM and LBM branches of different $\vec{q}$ points of the BL $\mathrm{MoS}_{2}$ unit-cell Brillouin zone will appear at the $\Gamma$ point of the MSL Brillouin zone. However, the projection of these folded modes onto BL $\mathrm{MoS}_{2} \mathrm{SM}$ and LBM eigenvectors at the $\Gamma$ point will be significantly smaller than that of the modes not arising due to zone folding. This is due to orthogonality of the eigenvectors at different $\vec{q}$ points. Therefore, considering the evolution of the eigenmodes with the largest projections (in fact, we consider all modes with $p^{\mathrm{SM}}, p^{\mathrm{LBM}}>0.1$ as SMs and LBMs), we infer the twist-angle dependence of shear and layer breathing modes. We can categorize the $\theta$ dependence of the low-frequency SM and LBM into three regions. Since the change of the low-frequency modes with twist angle is a result of complicated evolution of the interlayer coupling and mode mixing, we present both the frequencies and the eigenvectors of the SM and LBMs. The twist-angle dependence of the eigenvectors provides deeper insight into the evolution of the low-frequency modes, as we illustrate below.

\section{Region I: $\mathbf{7}^{\circ} \lesssim \theta \leqslant \mathbf{5 3}^{\circ}$}

In this region we find an averaged LBM and exceedingly small SM frequencies $\left(0-2 \mathrm{~cm}^{-1}\right.$, ultrasoft). The LBM frequency decreases monotonically as $\theta \rightarrow 7^{\circ}$ or $53^{\circ}$ from larger twists (Fig. 4). Furthermore, the projections $p^{\mathrm{LBM}}>0.9$ and $p^{\mathrm{SM}}>0.9$ indicate that the nature of vibrations of SM and LBMs remains similar to that of $\mathrm{BL} \mathrm{MoS}_{2}$. As a representative of this region, we show the eigenvectors corresponding to the SMs and LBMs in Figs. 5(c), 6(g), 6(h), 7(g), and 7(h) for $\theta=23.48^{\circ}$. Clearly, the SM (LBM) eigenvectors correspond to the relative horizontal (vertical) uniform displacement of the constituent layers. This is due to the absence of any extended high-symmetry stacking in the MSL, which leads to nearly uniform interlayer coupling. However, as we approach region II, the LBM starts mixing with in-plane modes giving rise to the monotonic decrease in the LBM frequencies. The change in LBM frequencies (by $\sim 1.5 \mathrm{~cm}^{-1}$ ) can be used to reliably infer large $\theta$. Moreover, the presence of the LBM also indicates that the layers in the twisted structures are not completely decoupled (in the out-of-plane direction).

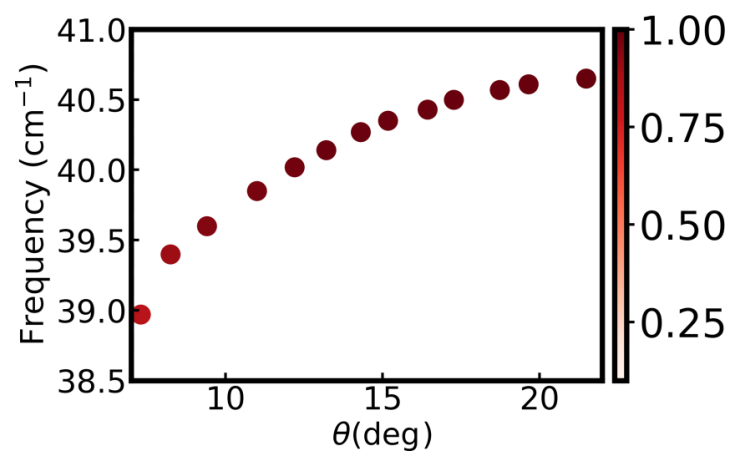

FIG. 4. Monotonic decrement of LBM frequencies of region I as $\theta \rightarrow 0^{\circ}$ in $\mathrm{TBL} \mathrm{MoS}_{2}$ with the color bar indicating $p^{\mathrm{LBM}}$. Identical results are obtained near $60^{\circ}$ as well. The calculated LBM frequency for $\mathrm{BL} \mathrm{MoS}_{2}$ is $43.5 \mathrm{~cm}^{-1}$. 


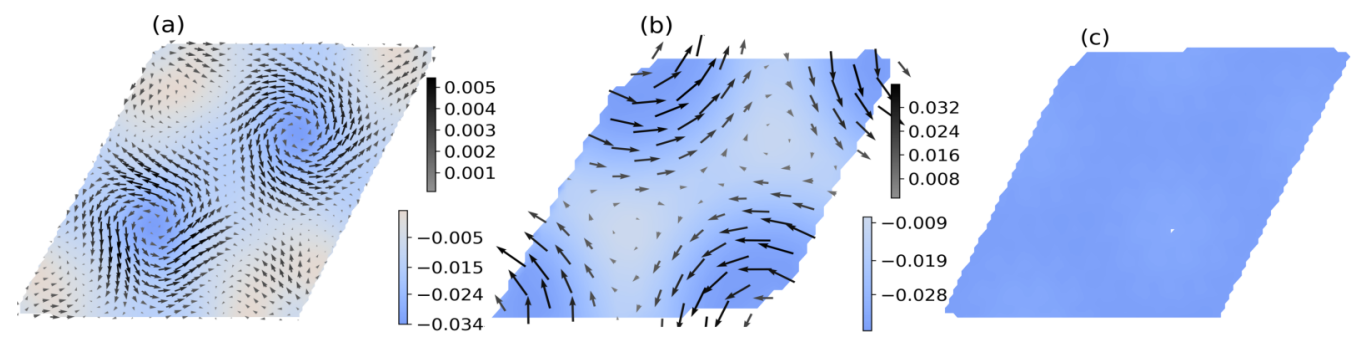

FIG. 5. Exact normalized eigenvectors with the largest projection on the bilayer LBM ( $\left.p^{\mathrm{LBM}}\right)$ for several values of $\theta$. The eigenvectors for the top Mo layer are for (a) $\theta=1.9^{\circ}$, (b) $\theta=5^{\circ}$, and (c) $\theta=23.48^{\circ}$. The corresponding bottom layers move exactly in the opposite direction. The arrows indicate the direction and magnitude of the in-plane displacement (with the associated color bar). The out-of-plane displacement is shown as a field (colored, with associated color bar). Only at large twist angles does the LBM resemble that of BL MoS 2 .

\section{2. $\operatorname{Region}$ II: $\mathbf{3}^{\circ} \lesssim \theta \lesssim \mathbf{7}^{\circ}$ and $\mathbf{5 3}^{\circ} \lesssim \theta \lesssim \mathbf{5 7}^{\circ}$}

We find that the LBM frequencies are quite sensitive to the twist angle including the presence of multiple LBMs. The eigenvectors corresponding to LBMs clearly indicate the mixing with in-plane modes. As an example, we show the LBM eigenvector with a maximum $p^{\mathrm{LBM}}$ for $\theta=5^{\circ}[\mathrm{Fig} .5(\mathrm{~b})]$. The in-plane modes corresponding to the LBM are of similar order of magnitude. In this region all the high-symmetry stackings and domain walls occupy a comparable area fraction of the MSL. This enhances the mode mixing. The ultrasoft SMs are also present in this region. However, the corresponding SM eigenvectors are no longer completely uniform [Figs. 6(e), 6(f), 7(e), and 7(f)] and start mixing with out-of-plane modes. In short, this region represents the transition from completely mismatched stacking (region I) to highly ordered stable stacking regions separated by domain walls (region III).

\section{Region III: $\theta \lesssim 3^{\circ}$ and $\theta \gtrsim 57^{\circ}$}

As $\theta$ decreases further $\left(\theta \rightarrow 0^{\circ}\right.$ or $\left.\theta \rightarrow 60^{\circ}\right)$ we find one LBM (with a significantly large $p^{\mathrm{LBM}}$ ) with frequency similar to that of stable BL MoS . This is due to the overwhelming growth of the stable stacking regions (Fig. 2) in this region.
The mixing of the LBM with in-plane modes exists, but the area is smaller compared to region II (Fig. 5). In order to establish this, we plot the LBM eigenvector with the largest $p^{\mathrm{LBM}}$ for $\theta=1.9^{\circ}$ [Fig. 5(a)]. It is evident from the figure that the LBM primarily arises from the out-of-plane vibration of the $A B$ stacked region. On the other hand, the SM frequencies split essentially into two branches: one ultrasoft in nature (similar to regions I and II) and one with high frequency (similar to $\mathrm{BL} \mathrm{MoS}_{2}, 22-28 \mathrm{~cm}^{-1}$ ). Moreover, as $\theta$ decreases from $3^{\circ}$ to $0^{\circ}\left(57^{\circ}\right.$ to $\left.60^{\circ}\right)$ the high-frequency SM redshifts [Figs. 3(d) and 3(e)]. The splitting of the SM has important consequences on the frictional properties, as we discuss later. The ultrasoft SMs are localized on domain walls and $A A$ stacking [domain walls and $A^{\prime} B$ near $60^{\circ}$, Figs. 6(a), 6(b), 7(a), and 7(b)]. On the contrary, high-frequency SMs primarily originate from the relative displacement of stable stacking regions [Figs. 6(c), 6(d), 7(c), and 7(d)]. The splitting of SM frequencies occurs due to significant growth of the stable stacking region in the TBL $\mathrm{MoS}_{2}$. The high-frequency SMs are quite similar in magnitude to $A B\left(A A^{\prime}\right)$. The apparent stiffening of these modes as $\theta \rightarrow 3^{\circ}$ is because of mixing with out-of-plane modes. For instance, the SM frequency of individual $A B$ stacking is $\sim 21 \mathrm{~cm}^{-1}$, whereas the high-frequency

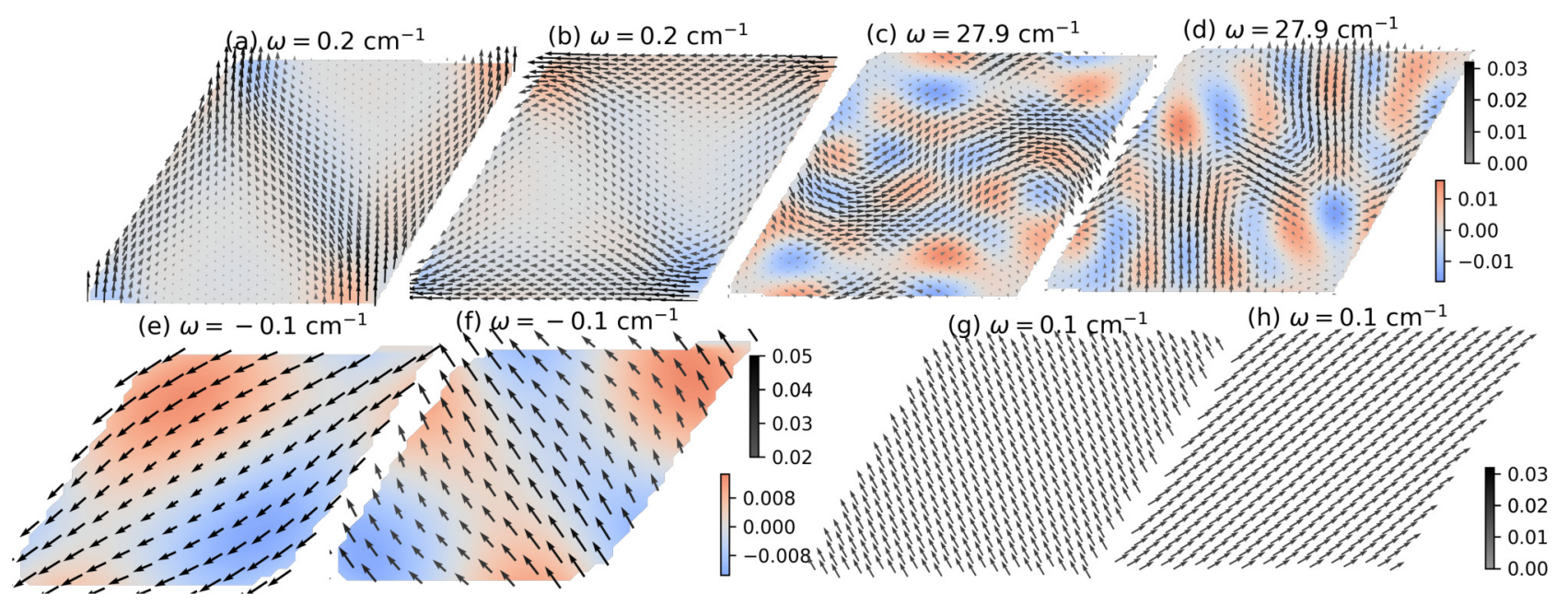

FIG. 6. Visualization of the eigenvectors at the $\Gamma$ point corresponding to the ultrasoft shear modes for (a) and (b) $\theta=1.9^{\circ}$, (e) and (f) $\theta=5^{\circ}$, and (g) and (h) $\theta=23.48^{\circ}$, along with (c) and (d) the high-frequency SMs. The positions of the domain walls and point defects are the same as shown in Fig. 1. The arrows (gray colorbar) denote in-plane displacements (only for Mo atoms of the top layer, for clarity), whereas out-of-plane displacements are represented as a continuous field (colored). The in-plane displacements of the Mo atoms of the bottom layer are exactly opposite to that of the top layer. 


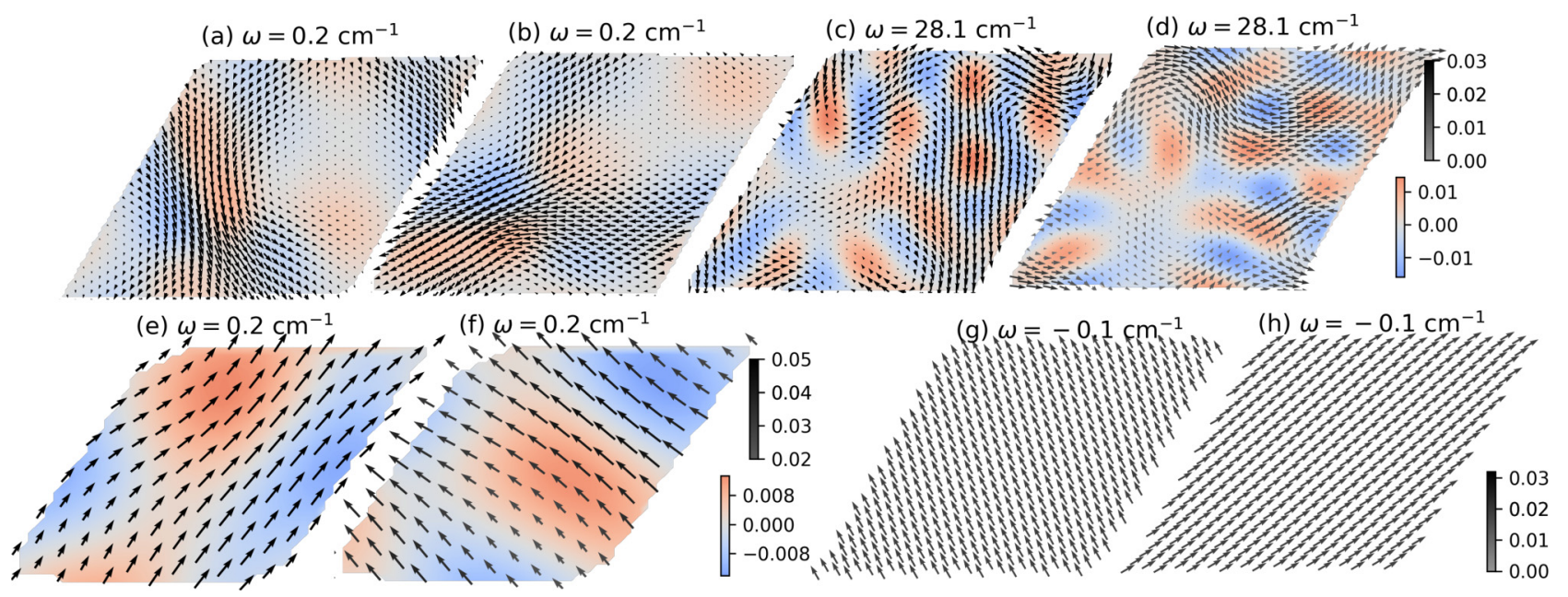

FIG. 7. Visualization of the eigenvectors at the $\Gamma$ point corresponding to the shear modes for (a) and (b) $\theta=58.1^{\circ}$, (e) and (f) $\theta=55^{\circ}$, and (g) and (h) $\theta=36.52^{\circ}$, along with (c) and (d) the high-frequency SMs. The position of the domain walls and point defects are the same as shown in Fig. 1. The eigenvectors are represented in similar manner as in Fig. 6.

SM of TBL $\mathrm{MoS}_{2}$ with $\theta=3^{\circ}$ is $\sim 28 \mathrm{~cm}^{-1}$. It should be pointed out again that the evolution of SMs does not arise from the folded modes of the unit-cell Brillouin zone as discussed earlier. The twist angle dependence of these eigenvectors is strongly dependent on the interlayer interaction strength. To further illustrate this point, we compare the ultrasoft SM eigenvectors for TBL $\mathrm{MoS}_{2}$ with $\theta=23.03^{\circ}$ (MSL lattice constant $\sim 47.3 \AA$ with 5418 atoms) and $\theta=1.9^{\circ}$ (MSL lattice constant $\sim 47.7 \AA$ with 5514 atoms). The ultrasoft SM eigenvectors in these two systems are remarkably different, although they are of similar dimensions. For $\theta=23.03^{\circ}$ the eigenvectors represent uniform relative displacement of the layers, whereas for $\theta=1.9^{\circ}$ the eigenvectors are localized. This shows that the eigenvectors corresponding to the ultrasoft SMs are controlled by the interlayer interaction strength and cannot be explained by simple zone-folding argument. Moreover, the high-frequency SMs are present only for $\theta=1.9^{\circ}$ and absent for $\theta=23.03^{\circ}$, further confirming our conclusion. The maximum variations in the SM and LBM frequencies $\left(\sim 8\right.$ and $\left.\sim 10 \mathrm{~cm}^{-1}\right)$ are comparable to those observed in Raman studies ( $\sim 8$ and $6.7 \mathrm{~cm}^{-1}$, respectively [29]). Such large variations of low-frequency modes and the appearance of multiple LBMs can be useful for characterization of bilayer properties.

\section{Ultrasoft shear modes: Phasons}

The presence of the ultrasoft modes with the twist angle is one of the major findings of our work. Thus, we investigate three important aspects of this finding: the origin of these modes, their twist-angle dependence, and consequences on frictional properties.

The ultrasoft modes represent an effective translation of the MSL by local relative displacements of the atoms in the constituent layers (see the Supplemental Material [49]). Since the frequencies associated with these modes are also very small (almost acoustic-mode-like), this implies that under the relative displacements of two layers (following the eigenvectors shown in Figs. 6 and 7) the energy of the TBL $\mathrm{MoS}_{2}$ remains invariant (or almost invariant), which can be a consequence of continuous symmetry breaking. For example, the in-plane acoustic modes (LA and TA modes), which represent the invariance of the total energy under global translation, originate due to translational symmetry breaking. However, strictly speaking, we show that the ultrasoft SMs found in our calculation are optical modes and do not represent continuous symmetry breaking. The optical nature is clearly reflected in the dispersion relation $(d \omega / d q \approx 0$ for small $q$ very near the $\Gamma$ point), unlike the acoustic modes [Fig. 8, small negative values at $\Gamma\left(\lesssim-0.2 \mathrm{~cm}^{-1}\right)$ are within numerical accuracies of our calculation]. We highlight both the acoustic and ultrasoft modes in order to show this difference clearly. The ultrasoft nature of these SMs can be understood from the one-dimensional Frenkel-Kontorova model. In this model, a linear chain of atoms is subjected to an external periodic

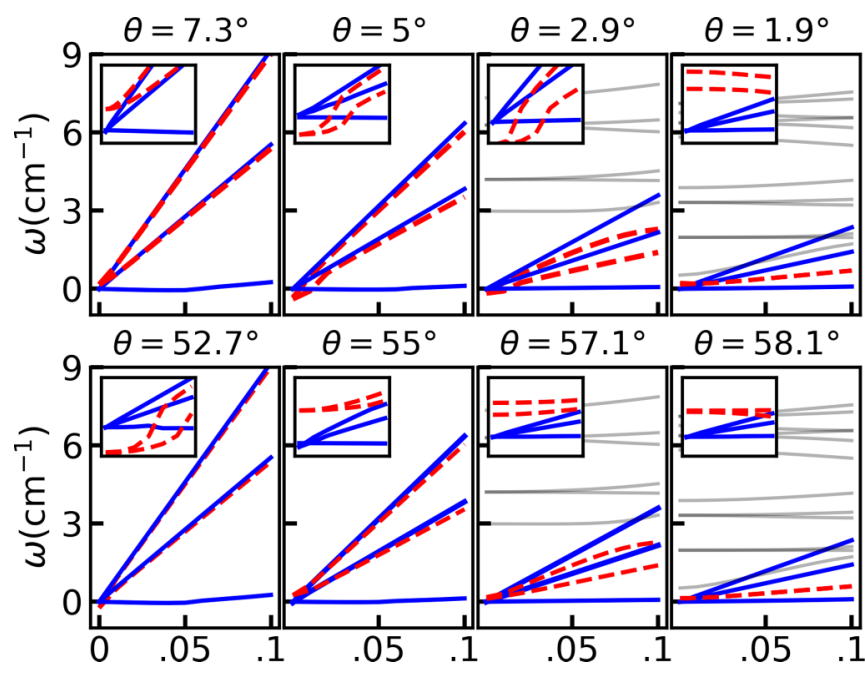

FIG. 8. Dispersion of the low-frequency modes for several values of $\theta$. The $x$ axis represents momentum, in units of $\frac{4 \pi}{\sqrt{3} a_{\mathrm{m}}}$, with $a_{\mathrm{m}}$ the moiré lattice constant. The LA, TA, and ZA (ultrasoft shear) modes are highlighted with blue solid (red dashed) lines. The insets show a close-up of dispersion for $q<0.005$ with $\omega<0.4 \mathrm{~cm}^{-1}$. 

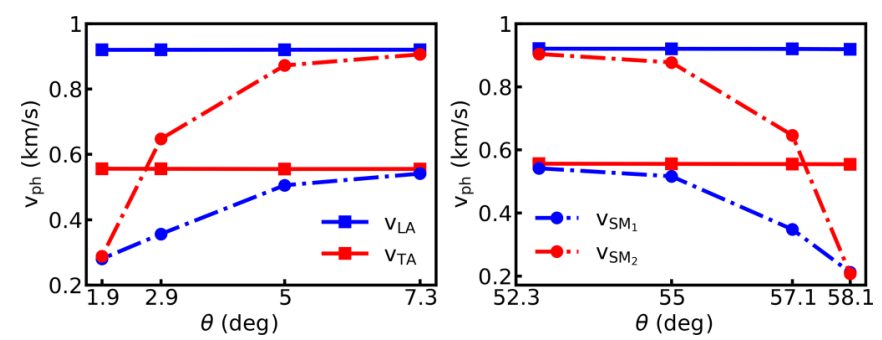

FIG. 9. Twist-angle dependence of the velocity of the phonon modes for both acoustic modes (LA and TA) and ultrasoft phason modes computed from the linear region of phonon dispersion.

potential. Depending on the ratio of the periodicity of the potential and linear chain, the structures can be commensurate or incommensurate. For simplicity, we assume the incommensurate (commensurate) structure as a lattice with infinite (finite and small) periodic length. Without considering dissipative coupling, the incommensurate structure possesses a gapless Goldstone mode (phason) with linear dispersion due to invariance of the phases of two mass density waves under uniform relative displacement. When a commensurate state is approached, the phason becomes gapped [50] with $d \omega / d q \approx$ 0 . This is exactly what happens in the case of ultrasoft SMs found in our calculations. The linear chain of atoms and the external periodic potential in the Frenkel-Kontorova model are replaced by the constituent $\mathrm{MoS}_{2}$ layers of TBL $\mathrm{MoS}_{2}$ and stacking-dependent binding energy, respectively. Since we only simulate commensurate angles, the phasons are always gapped (although ultrasoft) optical modes with $d \omega / d q \approx 0$. Hence, in our calculation the phase invariance associated with these modes is always approximate. In the case of incommensurate twist angles, however, the phase invariance becomes exact and we expect corresponding gapless phason modes with $\omega \propto q$.

The exact eigenvectors for the ultrasoft SMs cannot be directly probed by experiments. Keeping in mind the possible experimental signatures of the twist-angle dependence, we compute the $d \omega / d q$ (group velocity) of these ultrasoft modes. We compare them with the velocities of the acoustic modes (Fig. 9) away from the $\Gamma$ point using the linear dispersion of these modes. The velocity of the acoustic modes remains invariant with respect to the change of the twist angle, whereas the velocity of the ultrasoft phason modes changes significantly (by a factor of $2-3$ at $\theta=1.9^{\circ}, 58.1^{\circ}$ ). We also find that the dispersion of the low-frequency phonon modes are extremely sensitive to relaxation, particularly for small twist angles. Physically, the in-plane acoustic modes represent the long-wavelength vibrations of the entire twisted bilayer $\mathrm{MoS}_{2}$, with all the atoms of two layers participating. At the $\Gamma$ point, these modes correspond to uniform translation of all the atoms in the MSL. Therefore, the acoustic mode velocities within the harmonic approximation can be written in terms of the Lamé coefficients $\mu$ and $\lambda$ in the following manner: $v_{\mathrm{LA}}=\sqrt{(\lambda+2 \mu) / \rho}$ and $v_{\mathrm{TA}}=\sqrt{\mu / \rho}$ [50]. The presence of the MSL only affects the motion involving relative displacement of the constituent layers. Since the LA and TA modes only correspond to in-phase motion of the layers, they remain unaffected irrespective of the twist angle. Moreover, the rigidity (governed by $\lambda$ and $\mu$ ) of the lattice for the in-plane acoustic modes are the same as that of single-layer $\mathrm{MoS}_{2}$. This is evident from the twist-angle-independent behavior of the velocities of the LA and TA modes (Fig. 9). On the other hand, the ultrasoft phason modes are localized on domain walls and $A A$ stacking for $\theta \rightarrow 0^{\circ}$ (domain walls and $A^{\prime} B$ stacking). They represent acoustic modes of the emergent soft moirè scale lattice (containing only domain walls and $A A$ near $0^{\circ}$ and domain walls and $A^{\prime} B$ near $60^{\circ}$ ). Strictly speaking, they become acoustic modes only for incommensurate structures, as pointed out earlier. The soft nature of the lattice is noticeable from the change of velocity of these modes (Fig. 9). Similar to the LA and TA modes, the velocities of the phason modes are functions of $\lambda_{E}$ and $\mu_{E}$, where $\lambda_{E}$ and $\mu_{E}$ represent effective Lamé coefficients of the moirè scale lattice. The decrease in ultrasoft mode velocity implies a reduction in strength of the effective Lamé coefficients. This is because ultrasoft modes originate from the out-of-phase motion of the two layers. Thus, the strength of the interlayer coupling gives rise to effective Lamé coefficients. We leave details of the consequences of this emergent soft lattice and investigation of their rigidity for future work.

Before discussing the consequence of the twist-angle dependence of the ultrasoft SM frequencies on frictional properties, we briefly summarize previous experimental studies of this aspect. The introduction of a twist between two layers of two-dimensional materials has been shown to modify the frictional properties dramatically [24-26,51-57]. Depending on the twist angle, the frictional force can be exceedingly small (structural superlubricity) or moderately large. For instance, the frictional force can drop by two to three orders of magnitude if the twist angle is greater than $5^{\circ}$ or less than $55^{\circ}$ in the case of graphene on graphite, twisted bilayer $\mathrm{MoS}_{2}[24,51,53,58]$. Also, for $5^{\circ} \lesssim \theta \lesssim 55^{\circ}$, the frictional properties remain almost constant. In all these examples, maximum friction is obtained when the two layers are untwisted. Although microscopic details of the interlayer sliding process giving rise to superlubric behavior can be quite involved, the primary reason behind superlubricity is often attributed to incommensurability between two surfaces. Here we identify that the change of frictional properties with $\theta$ is intimately related to the existence and evolution of the ultrasoft modes. For $\theta=0^{\circ}$ or $60^{\circ}$, the $\mathrm{BL} \mathrm{MoS}_{2}$ is unit-cell commensurate. When trying to shear one layer with respect to another in $\mathrm{BL} \mathrm{MoS}_{2}$, all unit cells have to cross the interlayer-sliding barrier simultaneously. This leads to large SM frequencies $\left(\sim 21 \mathrm{~cm}^{-1}\right)$ and high friction. This can also be understood from the aforementioned one-dimensional Frenkel-Kontorova model. In the unit-cell commensurate case [Fig. 10(a)], while shearing the atoms globally with respect to the external periodic potential, every atom rises toward the hill. Thus, the cost of shearing is large. However, if the system is largescale periodic [Fig. 10(b)], then every atom has to cross a variable sliding barrier. This reduces the shearing energy, due to cancellation of cost while ascending the hill and gain while descending it. Similarly, TBL $\mathrm{MoS}_{2}$ for large twist angles $\left(\theta \lesssim 5^{\circ}, \gtrsim 55^{\circ}\right)$ is periodic at larger scale where shearing unit cells have to cross a variable interlayer-sliding barrier (due to the coexistence of multiple stackings with different binding energies). In effect, this drastically reduces SM 
(a)

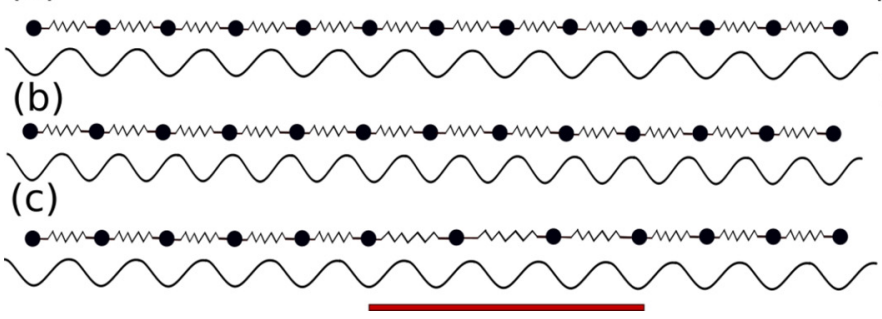

(d)

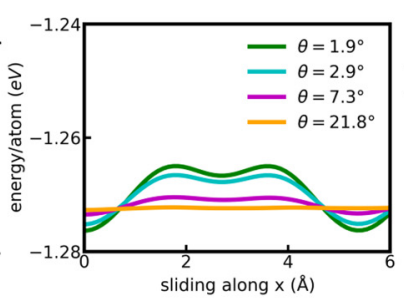

(e)

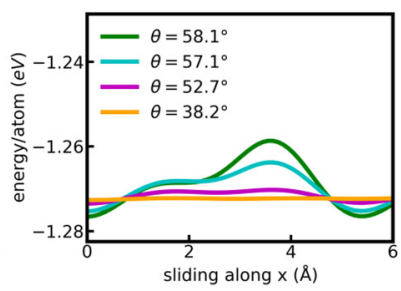

FIG. 10. One-dimensional Frenkel-Kontorova model with (a) unit-cell commensuration, (b) large-scale commensuration, and (c) mostly unit-cell commensurate with discommensuration in between. The discommensuration is marked with a red line. Also shown is the development of a barrier against shearing as (d) $\theta \rightarrow 0^{\circ}$ and (e) $\theta \rightarrow 60^{\circ}$. After relaxation of the twisted structures we slide the top MoS $\mathrm{S}_{2}$ layer along the $x$ axis and calculate the total energy without further relaxation.

frequencies and hence friction as well (superlubric). This can be easily confirmed from the existence of the ultrasoft phason modes and the absence of high-frequency SMs. However, as $\theta$ decreases further, not only do the high-frequency SMs similar to the untwisted bilayer appear, but also the ultrasoft modes start to localize on the domain walls (splitting of SMs). This immediately implies that to globally shear one layer with respect to the other, a large barrier has to be overcome. Because only a small fraction of atoms participate in the ultrasoft modes in this case, unlike large twist angle, a significant fraction of atoms participate in the high-frequency SM, implying pinning. The origin of the pinning lies in the significant growth of stable stacking when $\theta \rightarrow 0^{\circ}$ or $60^{\circ}$ (Fig. 2), leading to the development of a large interlayer sliding barrier against shearing [Figs. 10(d) and 10(e)]. Similarly, if the potential is strong enough in the Frenkel-Kontorova model, most atoms sit at the minima. Only a small number of atoms occupy the energetically unfavorable hills, known as discommensuration [Fig. 10(c)]. This leads to pinned phasons in the Frenkel-Kontorova incommensurate structures. The nature of vibrations of ultrasoft phason modes in our calculations (uniform at large $\theta$ due to complete stacking mismatch and nonuniform at small $\theta$ due to overwhelming growth of stable stacking) indicates the possibility of having pinned phasons (Aubry-like transition) in the small twist incommensurate structures [50,59-61]. Interestingly, similar pinning behavior has also been realized in systems such as colloidal monolayers in optical lattices $[62,63]$ and physisorbed submonolayers on crystal surfaces [64].

\section{DISCUSSION}

\section{A. Other 2D materials}

Twisting one layer with respect to another in bilayers of 2D materials leads to the presence of multiple types of stacking in the MSL with different binding energies and stability, irrespective of the material's electronic properties. The intralayer interaction in 2D materials is far stronger than the interlayer coupling. The combination of strong in-plane stiffness and weak variable interlayer coupling of twisted structures should produce similar behavior of low-frequency vibrational modes in any 2D material. Thus, the existence of ultrasoft phason modes, the twist-angle dependence of the corresponding eigenvectors and velocity, is expected to be generic to any 2D materials. The twist-angle dependence of the interlayer coupling is also revealed in the relaxed twisted structures. In order to illustrate, we compute the low-frequency vibrational modes for twisted bilayer $\mathrm{MoSe}_{2}$ using the MVACF at $T=$ $300 \mathrm{~K}$ (Fig. 11). The trends are quite similar to the case of TBL $\mathrm{MoS}_{2}$, justifying our conclusion.

\section{B. Raman spectroscopy}

We have demonstrated the evolution of shear and layer breathing mode frequencies in twisted TMD bilayers. Irrespective of their Raman sensitivity, these modes are present in the twisted bilayer structures. For BL $\mathrm{MoS}_{2}$ (untwisted, most stable stacking), both the SM and LBM are found to be Raman active [28]. The observation of these modes using Raman spectroscopy depends on the Raman scattering intensity [28]. Since both the SM and LBM in the case of BL $\mathrm{MoS}_{2}$ are Raman active, we expect in the twisted structures that the SM and LBM with the largest $p^{\mathrm{SM}}$ and $p^{\mathrm{LBM}}$ will also be Raman active. However, this is speculative and explicit calculations of Raman intensity in twisted structures are unfeasible at present. Hence, we compare our results directly to Raman measurements in the case of TBL $\mathrm{MoSe}_{2}$ to show the usefulness of our calculations in Fig. 12. The trends of the twist-angle dependence of low-frequency vibrational modes

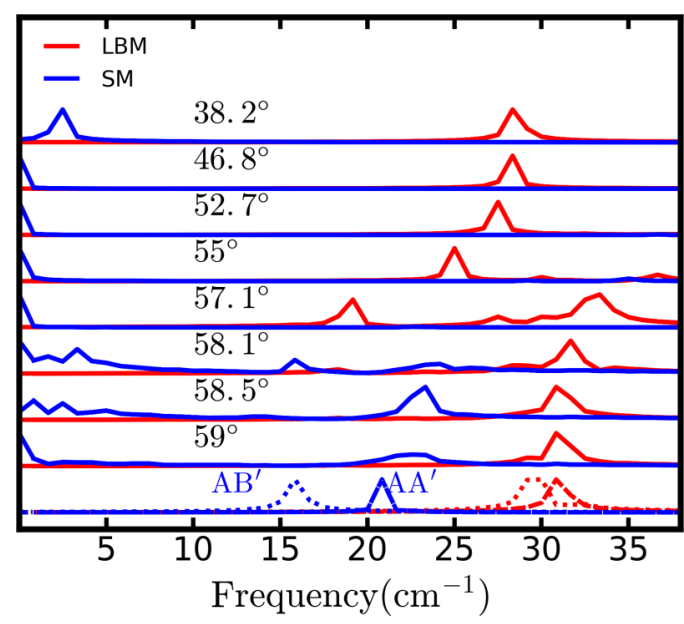

FIG. 11. Evolution of low-frequency vibrational modes with twist angles for a twisted bilayer of $\mathrm{MoSe}_{2}$. The evolution of lowfrequency modes as $\theta \rightarrow 0^{\circ}$ is similar to that as $\theta \rightarrow 60^{\circ}$ and hence is not shown here. 


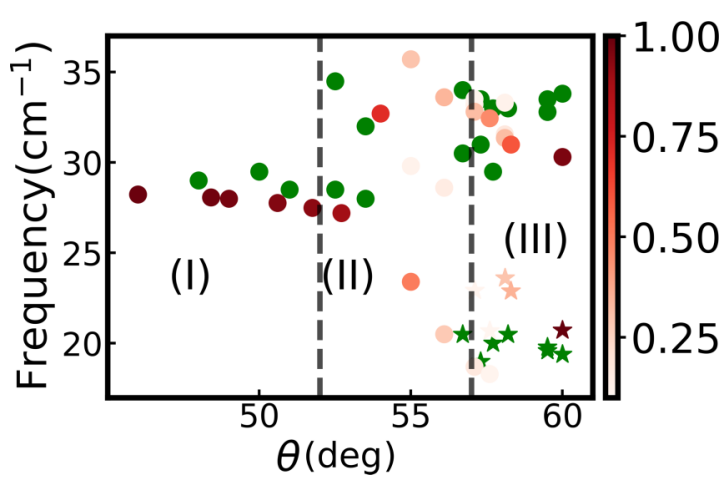

FIG. 12. Comparison of the calculated twist-angle dependence of low-frequency vibrational modes and Raman measurements [30] in TBL MoSe $e_{2}$. The experimental SMs (LBMs) are denoted by green stars (circles), whereas the computed SMs (LBMs) are denoted by variable red stars (circles). The color bar indicates $p^{\mathrm{SM}}$ and $p^{\mathrm{LBM}}$.

in TBL $\mathrm{MoSe}_{2}$ are in excellent agreement with experiment [30]. Similar to TBL $\mathrm{MoS}_{2}$, we categorize the $\theta$ dependence of SM and LBM frequencies. In region III, the high-frequency SM appears and LBM frequencies are similar to that of BL $\mathrm{MoSe}_{2}$, as predicted by our calculations. Multiple LBMs and significant variation in LBM frequencies are also present in region II. In both regions I and II the SM frequencies are absent (because they are ultrasoft in nature) in Raman spectroscopy. However, two prominent features seem to be missing in the Raman experiment [30]: (i) monotonic decrement of LBM frequencies in region I (as in Fig. 4) and (ii) slight stiffening of the SM frequencies compared to $\mathrm{BL} \mathrm{MoSe}_{2}$ (region III). This might be due to the fact that the experiment is carried out with chemical vapor deposition grown samples, which is known to exhibit less mobility and more disorder. We expect samples of better quality, such as mechanically exfoliated structures that can show these missing features.

\section{Brillouin-Mandelstam spectroscopy}

Due to ultrasoft nature $\left(\lesssim 1 \mathrm{~cm}^{-1}\right)$ of the phason modes, Raman spectroscopy cannot be used to probe them. BrillouinMandelstam spectroscopy (BMS), which can probe small frequencies (typically $0.1-6 \mathrm{~cm}^{-1}$ ), is often used in mineral physics and material science to probe acoustic modes [65]. The phonon dispersion can also be mapped out by changing the incident light angle in BMS [66]. Therefore, the sound speed along with elastic rigidity can be experimentally obtained. Using BMS, our predictions of the existence of the ultrasoft modes can be verified. Also, by measuring the twist-angle dependence of the dispersion of the acoustic and ultrasoft modes, our results on the velocity dependence of ultrasoft modes can be tested. However, it is more likely to find incommensurate twisted structures in experiments and thus the phason modes may become completely gapless. Still, we expect the phason velocity to be twist-angle dependent as predicted here.

\section{More twistnonics}

We have demonstrated the manipulation of low-frequency vibrational modes with twist and related effects in frictional

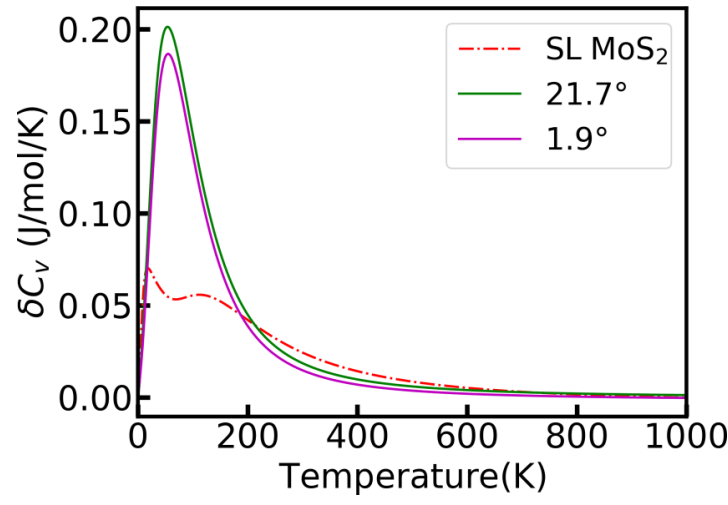

FIG. 13. Twist-angle dependence difference in specific heat (in units of $\mathrm{J} / \mathrm{mol} / \mathrm{K}$ ) of $\mathrm{TBL} \mathrm{MoS}_{2}$ with respect to $\mathrm{BL} \mathrm{MoS}_{2}$ (untwisted).

properties in transition-metal dichalcogenide bilayers. Since the phonon plays an important role in determining many other material properties, the ability to control the phonon dispersion will facilitate engineering of those properties [67]. Here we outline a few of them. (i) The existence of ultrasoft modes can strongly modify the low-temperature specific heat $[68,69]$, one of the key variables that dictates thermodynamic properties of a material. In order to illustrate this, we calculate the difference in specific heat of twisted structures with respect to $\mathrm{BL} \mathrm{MoS}_{2}$ (Fig. 13). Clearly, there are significant changes in $\delta C_{v}$ for $T<200 \mathrm{~K}$. The corresponding single-layer $\mathrm{MoS}_{2}$ value is also shown. (ii) Acoustic phonons are known to be the dominant heat carriers in insulating materials. The presence of ultrasoft phason modes can significantly modify the thermal conductivity of the twisted structures. Furthermore, as the group velocities of the ultrasoft phason modes can be tuned with twist angles, the thermal conductivity can also be engineered. (iii) The electron-phonon coupling for the ultrasoft modes can play an important role in determining electrical resistivity. For example, in the case of twisted bilayer graphene, electron-acoustic phonon coupling has been identified as the dominant source of its high- $T$ resistivity behavior [70]. The authors assumed the relative displacement of the two layers in twisted bilayer graphene as the additional acoustic modes of the system with identical acoustic mode (LA and TA) dispersion. Although, this model captures the features of the resistivity qualitatively, the $D / v_{\text {ph }}(D$ is deformation potential for the electron-acoustic phonon coupling, $v_{\mathrm{ph}}$ is the phonon velocity) appearing in theoretical calculations seems to be off by a factor of 2-3 compared to experiment $[70,71]$. The assumption of the shear modes as the acoustic modes is reasonable in the twisted structures, as they are ultrasoft in nature. However, the assumption that the dispersions of the ultrasoft modes and acoustic modes are identical is not accurate. Our calculations for the TBL $\mathrm{MoS}_{2}$ clearly show the twist-angle dependence of the velocity of the phason modes compared to that of the acoustic modes (differing by a factor of 2-3). This might provide an explanation for the missing factor in the case of TBLG.

Since the SM and LBM are interlayer coupling dependent, by modifying the interlayer interaction strength (with external 
pressure, for example) the phonon spectra can be further engineered. Another important degree of freedom to tune phononic properties is the choice of materials, for instance, van der Waals heterostructures (stacking of two dissimilar 2D materials).

\section{CONCLUSION}

Here we have shown that the low-frequency modes are extremely sensitive to twisting in the twisted TMD bilayer and can be used as a probe to determine the twist angle. We have also made predictions about the presence of ultrasoft phason modes and the twist-angle dependence of their eigenvectors and velocities. Our results indicate a twist-angle-dependent transition from a superlubric to a pinned state. Our study provides a step towards twistnonics in 2D materials.

Note added. Recently, we become aware of two recent studies, carried out within an elastic continuum approximation, reporting the existence of similar ultrasoft phonon modes in TBLG $[72,73]$.

\section{ACKNOWLEDGMENTS}

The authors thank Rahul Debnath, Shinjan Mandal, Sriram Ramaswamy, Sumilan Banerjee, Subroto Mukerjee, Arindam Ghosh, and Rahul Pandit for useful discussions and thank the Supercomputer Education and Research Center at IISc for providing computational resources.
[1] J. S. Alden, A. W. Tsen, P. Y. Huang, R. Hovden, L. Brown, J. Park, D. A. Muller, and P. L. McEuen, Proc. Natl. Acad. Sci. USA 110, 11256 (2013).

[2] H. Yoo, R. Engelke, S. Carr, S. Fang, K. Zhang, P. Cazeaux, S. H. Sung, R. Hovden, A. W. Tsen, T. Taniguchi, K. Watanabe, G.-C. Yi, M. Kim, M. Luskin, E. B. Tadmor, E. Kaxiras, and P. Kim, Nat. Mater. 18, 448 (2019).

[3] S. S. Sunku, G. X. Ni, B. Y. Jiang, H. Yoo, A. Sternbach, A. S. McLeod, T. Stauber, L. Xiong, T. Taniguchi, K. Watanabe, P. Kim, M. M. Fogler, and D. N. Basov, Science 362, 1153 (2018).

[4] S. Dai, Y. Xiang, and D. J. Srolovitz, Nano Lett. 16, 5923 (2016).

[5] K. Zhang and E. B. Tadmor, J. Mech. Phys. Solids 112, 225 (2018).

[6] F. Gargiulo and O. V. Yazyev, 2D Mater. 5, 015019 (2018).

[7] S. Huang, K. Kim, D. K. Efimkin, T. Lovorn, T. Taniguchi, K. Watanabe, A. H. MacDonald, E. Tutuc, and B. J. LeRoy, Phys. Rev. Lett. 121, 037702 (2018).

[8] I. V. Lebedeva and A. M. Popov, Phys. Rev. B 99, 195448 (2019).

[9] S. Shallcross, S. Sharma, and O. A. Pankratov, Phys. Rev. Lett. 101, 056803 (2008)

[10] S. Shallcross, S. Sharma, E. Kandelaki, and O. A. Pankratov, Phys. Rev. B 81, 165105 (2010).

[11] N. N. T. Nam and M. Koshino, Phys. Rev. B 96, 075311 (2017).

[12] M. Angeli, E. Tosatti, and M. Fabrizio, Phys. Rev. X 9, 041010 (2019).

[13] R. Bistritzer and A. H. MacDonald, Proc. Natl. Acad. Sci. USA 108, 12233 (2011).

[14] Y. W. Choi and H. J. Choi, Phys. Rev. B 98, 241412(R) (2018).

[15] G. Tarnopolsky, A. J. Kruchkov, and A. Vishwanath, Phys. Rev. Lett. 122, 106405 (2019).

[16] Y. Cao, V. Fatemi, A. Demir, S. Fang, S. L. Tomarken, J. Y. Luo, J. D. Sanchez-Yamagishi, K. Watanabe, T. Taniguchi, E. Kaxiras et al., Nature (London) 556, 80 (2018).

[17] Y. Cao, D. Chowdhury, D. Rodan-Legrain, O. Rubies-Bigordà, K. Watanabe, T. Taniguchi, T. Senthil, and P. Jarillo-Herrero, Phys. Rev. Lett. 124, 076801 (2020).

[18] Y. Cao, V. Fatemi, S. Fang, K. Watanabe, T. Taniguchi, E. Kaxiras, and P. Jarillo-Herrero, Nature (London) 556, 43 (2018).

[19] S. Carr, D. Massatt, S. Fang, P. Cazeaux, M. Luskin, and E. Kaxiras, Phys. Rev. B 95, 075420 (2017).
[20] A. Kerelsky, L. McGilly, D. M. Kennes, L. Xian, M. Yankowitz, S. Chen, K. Watanabe, T. Taniguchi, J. Hone, C. Dean, A. Rubio, and A. N. Pasupathy, Nature (London) 572, 95 (2019).

[21] P. Lucignano, D. Alfè, V. Cataudella, D. Ninno, and G. Cantele, Phys. Rev. B 99, 195419 (2019).

[22] X. Lu, P. Stepanov, W. Yang, M. Xie, M. A. Aamir, I. Das, C. Urgell, K. Watanabe, T. Taniguchi, G. Zhang et al., Nature (London) 574, 653 (2019).

[23] G. Trambly de Laissardière, D. Mayou, and L. Magaud, Nano Lett. 10, 804 (2010).

[24] M. Dienwiebel, G. S. Verhoeven, N. Pradeep, J. W. M. Frenken, J. A. Heimberg, and H. W. Zandbergen, Phys. Rev. Lett. 92 , 126101 (2004).

[25] O. Hod, Phys. Rev. B 86, 075444 (2012).

[26] W. Wang, J. Shen, and Q.-C. He, Phys. Rev. B 99, 054103 (2019).

[27] I. Maity, P. K. Maiti, and M. Jain, Phys. Rev. B 97, 161406(R) (2018).

[28] Y. Zhao, X. Luo, H. Li, J. Zhang, P. T. Araujo, C. K. Gan, J. Wu, H. Zhang, S. Y. Quek, M. S. Dresselhaus, and Q. Xiong, Nano Lett. 13, 1007 (2013).

[29] S. Huang, L. Liang, X. Ling, A. A. Puretzky, D. B. Geohegan, B. G. Sumpter, J. Kong, V. Meunier, and M. S. Dresselhaus, Nano Lett. 16, 1435 (2016).

[30] A. A. Puretzky, L. Liang, X. Li, K. Xiao, B. G. Sumpter, V. Meunier, and D. B. Geohegan, ACS Nano 10, 2736 (2016).

[31] A. I. Cocemasov, D. L. Nika, and A. A. Balandin, Phys. Rev. B 88, 035428 (2013).

[32] M. H. Naik, I. Maity, P. K. Maiti, and M. Jain, J. Phys. Chem. C 123, 9770 (2019).

[33] M.-L. Lin, Q.-H. Tan, J.-B. Wu, X.-S. Chen, J.-H. Wang, Y.-H Pan, X. Zhang, X. Cong, J. Zhang, W. Ji, P.-A. Hu, K.-H. Liu, and P.-H. Tan, ACS Nano 12, 8770 (2018).

[34] M. H. Naik and M. Jain, Phys. Rev. Lett. 121, 266401 (2018).

[35] M. Fleischmann, R. Gupta, S. Sharma, and S. Shallcross, arXiv:1901.04679

[36] F. Wu, T. Lovorn, E. Tutuc, I. Martin, and A. H. MacDonald, Phys. Rev. Lett. 122, 086402 (2019).

[37] L. An, X. Cai, M. Huang, Z. Wu, J. Lin, Z. Ying, Z. Ye, X. Feng, and N. Wang, arXiv:1907.03966.

[38] S. Plimpton, J. Comput. Phys. 117, 1 (1995). 
[39] http://lammps.sandia.gov.

[40] E. Bitzek, P. Koskinen, F. Gähler, M. Moseler, and P. Gumbsch, Phys. Rev. Lett. 97, 170201 (2006).

[41] J.-W. Jiang, Nanotechnology 26, 315706 (2015).

[42] J.-W. Jiang and Y.-P. Zhou, Handbook of Stillinger-Weber Potential Parameters for Two-Dimensional Atomic Crystals (IntechOpen, 2017).

[43] A. N. Kolmogorov and V. H. Crespi, Phys. Rev. B 71, 235415 (2005).

[44] A. Togo and I. Tanaka, Scr. Mater. 108, 1 (2015)

[45] https://github.com/abelcarreras/phonolammps.

[46] D.-B. Zhang, T. Sun, and R. M. Wentzcovitch, Phys. Rev. Lett. 112, 058501 (2014).

[47] E. N. Koukaras, G. Kalosakas, C. Galiotis, and K. Papagelis, Sci. Rep. 5, 12923 (2015).

[48] S. Carr, D. Massatt, S. B. Torrisi, P. Cazeaux, M. Luskin, and E. Kaxiras, Phys. Rev. B 98, 224102 (2018).

[49] See Supplemental Material at http://link.aps.org/supplemental/ 10.1103/PhysRevResearch.2.013335 for movies that represent effective translation of moiré lattices due to ultrasoft shear modes.

[50] P. M. Chaikin and T. C. Lubensky, Principles of Condensed Matter Physics (Cambridge University Press, Cambridge, 1995), Vol. 1.

[51] R. Ribeiro-Palau, C. Zhang, K. Watanabe, T. Taniguchi, J. Hone, and C. R. Dean, Science 361, 690 (2018).

[52] E. Koren and U. Duerig, Phys. Rev. B 93, 201404(R) (2016).

[53] H. Li, J. Wang, S. Gao, Q. Chen, L. Peng, K. Liu, and X. Wei, Adv. Mater. 29, 1701474 (2017).

[54] E. Koren and U. Duerig, Phys. Rev. B 94, 045401 (2016).

[55] I. Leven, D. Krepel, O. Shemesh, and O. Hod, J. Phys. Chem. Lett. 4, 115 (2012).

[56] M. R. Vazirisereshk, H. Ye, Z. Ye, A. Otero-de-la Roza, M.-Q. Zhao, Z. Gao, A. T. C. Johnson, E. R. Johnson, R. W. Carpick, and A. Martini, Nano Lett. 19, 5496 (2019).
[57] V. E. Claerbout, T. Polcar, and P. Nicolini, Comput. Mater. Sci. 163, 17 (2019).

[58] T. Onodera, Y. Morita, R. Nagumo, R. Miura, A. Suzuki, H. Tsuboi, N. Hatakeyama, A. Endou, H. Takaba, F. Dassenoy et al., J. Phys. Chem. B 114, 15832 (2010).

[59] T. C. Lubensky, S. Ramaswamy, and J. Toner, Phys. Rev. B 32, 7444 (1985).

[60] M. Peyrard and S. Aubry, J. Phys. C 16, 1593 (1983).

[61] P. Bak, Rep. Prog. Phys. 45, 587 (1982).

[62] T. Brazda, A. Silva, N. Manini, A. Vanossi, R. Guerra, E. Tosatti, and C. Bechinger, Phys. Rev. X 8, 011050 (2018).

[63] D. Mandelli, A. Vanossi, M. Invernizzi, S. Paronuzzi, N. Manini, and E. Tosatti, Phys. Rev. B 92, 134306 (2015).

[64] M. Pierno, L. Bruschi, G. Mistura, G. Paolicelli, A. Di Bona, S. Valeri, R. Guerra, A. Vanossi, and E. Tosatti, Nat. Nanotechnol. 10, 714 (2015).

[65] S. Speziale, H. Marquardt, and T. S. Duffy, Rev. Mineral. Geochem. 78, 543 (2014).

[66] F. Kargar, B. Debnath, J.-P. Kakko, A. Säynätjoki, H. Lipsanen, D. L. Nika, R. K. Lake, and A. A. Balandin, Nat. Commun. 7, 13400 (2016).

[67] A. A. Balandin, J. Nanosci. Nanotechnol. 5, 1015 (2005).

[68] H. Li, H. Ying, X. Chen, D. L. Nika, A. I. Cocemasov, W. Cai, A. A. Balandin, and S. Chen, Nanoscale 6, 13402 (2014).

[69] D. L. Nika, A. I. Cocemasov, and A. A. Balandin, Appl. Phys. Lett. 105, 031904 (2014).

[70] F. Wu, E. Hwang, and S. Das Sarma, Phys. Rev. B 99, 165112 (2019).

[71] H. Polshyn, M. Yankowitz, S. Chen, Y. Zhang, K. Watanabe, T. Taniguchi, C. R. Dean, and A. F. Young, Nat. Phys. 15, 1011 (2019).

[72] M. Koshino and Y.-W. Son, Phys. Rev. B 100, 075416 (2019).

[73] H. Ochoa, Phys. Rev. B 100, 155426 (2019). 